\title{
2D biological representations with reduced speckle obtained from two perpendicular ultrasonic arrays
}

\author{
Miguel A. Rodriguez-Hernandez ${ }^{\mathrm{a}, *}$, Angel Gomez-Sacristan ${ }^{\mathrm{a}}$ and Víctor M. Sempere-Payáb \\ aTACA, Universitat Politècnica de València, Valencia, Spain \\ ${ }^{\mathrm{b}}$ ITI, Universitat Politècnica de València, Valencia, Spain
}

\begin{abstract}
Ultrasound diagnosis is a widely used medical tool. Among the various ultrasound techniques, ultrasonic imaging is particularly relevant. This paper presents an improvement to a two-dimensional (2D) ultrasonic system using measurements taken from perpendicular planes, where digital signal processing techniques are used to combine one-dimensional (1D) A-scans were acquired by individual transducers in arrays located in perpendicular planes. An algorithm used to combine measurements is improved based on the wavelet transform, which includes a denoising step during the 2D representation generation process. The inclusion of this new denoising stage generates higher quality 2D representations with a reduced level of speckling. The paper includes different 2D representations obtained from noisy A-scans and compares the improvements obtained by including the denoising stage.
\end{abstract}

Keywords: 2D representation, perpendicular arrays, ultrasonics, denoising, wavelets

\section{Introduction}

Ultrasonic imaging is a commonly used tool in medical diagnosis. Image quality, resolution and contrast largely depend on the degree of speckling. This speckle or noise is due to the reflection of the ultrasounds by the tissue's microstructure. It is non-stationary and has a high statistical correlation with the signal that is being sought for. The reduction of speckle in ultrasonic images has been widely discussed, and different methods based on spatial or frequency diversity have been proposed [1].

Two-dimensional (2D) ultrasonic images (B-scans) are formed from one-dimensional (1D) traces (Ascans), and collected by different transducers that emit and receive radiofrequency (RF) signals. Many of the techniques that have been proposed to reduce speckle work directly with B-scans [2-4], although there are others that focus directly on the denoising of RF signals $[5,6]$. For example, denoising of the RF signals is an important step when Doppler techniques are used $[7,8]$.

Different tools have been used to reduce speckling. Wavelet transform has been used in different fields including denoising ultrasonic signals. The basic technique, based on the discrete wavelet transform (DWT), consists of calculating the DWT of the noisy register followed by the thresholding of wavelet coefficients and concluding with the recovery of the original register without noise by performing the

\footnotetext{
${ }^{*}$ Corresponding author: Miguel A. Rodriguez-Hernandez, ITACA, Universitat Politècnica de València, Camino Vera s/n, 46022 Valencia, Spain. E-mail: marodrig@ upvnet.upv.es.
} 
inverse DWT [3,4,9-13]. DWT is not a shift variant transform that can produce different results depending on the phase. The undecimated wavelet transform (UWT) is used to avoid this dependence. UWT is a shift invariant transform and produces improved results in denoising applications [14-17]. Moreover, UWT maintains the temporal information of the initial trace.

The use of several ultrasonic transducers provides complementary information on the tissue under diagnosis [18-22]. The transducers can be located in the same plane or in different planes. There are a number of monitoring schemes proposed in the literature, and this work is based on a system that contains two perpendicular linear ultrasound arrays, with a small number of transducers. The transducers emit in a sequential way, work in near-field with collimated beams and use the pulse-echo technique to obtain A-scans. These A-scans must be combined using digital signal processing techniques to obtain useful 2D representations of the areas under observation [21,22].

Although these digital signal processing techniques produce improved signal to noise ratios (SNRs) compared with the SNRs of the initial A-scans [21,22], the quality of the 2D representations is limited, especially by speckles. This $2 \mathrm{D}$ speckle depends on the combination signal processing method chosen and the initial noise in the A-scans. This paper proposes an improvement in the method used to generatse the 2D representations from a very limited set of A-scans obtained by perpendicular arrays in the near field and based on the wavelet transform [22]. The improvement consists of performing denoising simultaneously during the fusion of the A-scans. Thus, wavelet transform has a double function: to perform the fusion as in Ref. [21] and to carry out the denoising by simultaneously thresholding the wavelet coefficients. The 2D representations obtained using this new method have a superior quality to those obtained by the method described in Ref. [21], especially when the initial A-scans contain high levels of noise.

The objective of this work is to demonstrate that the use of the denoising wavelet methods applied to A-scans during the fusion process described in Ref. [21] produces 2D biological representations with improved quality. The main contribution of this research is the application of wavelet denoising techniques to the fusion process described in Ref. [21].

This paper is set out in the following manner. Section 2 describes the method used to combine the Ascans using the wavelet. This method includes the denoising step. Section 3 describes the experimental setup and discusses about the results obtained using different parameters. The conclusions are described at the end of the paper.

\section{Combination method}

The method starts with the acquisition of the ultrasonic measurements taken by the different transducers. Each of the two perpendicular arrays will have $N$ transducers; therefore the number of A-scans to be combined will be $2 N$. Each transducer emits a pulse and receives the corresponding A-scan to be combined sequentially. Figure 1 shows a schematic representation of A-scans acquisition process. The following nomenclature will be used for the A-scans in order to distinguish each of the measurements made by each transducer:

$$
X_{I}(t)
$$

where $X$ can be $V$ (vertical) or $H$ (horizontal), $I$ is the transducer number $(1 \ldots \mathrm{N})$ and $t$ is the time. Therefore, for example, $V_{3}(t)$ represents the A-scan taken by vertical transducer number 3 .

The second step consists of obtaining the wavelet transform of each of the $N$ A-scans measured in the previous step. The transform used is the Undecimated Wavelet Packet Transform (UWPT). The undecimated transform variant makes the transform shift independent and ensures that the new registers 


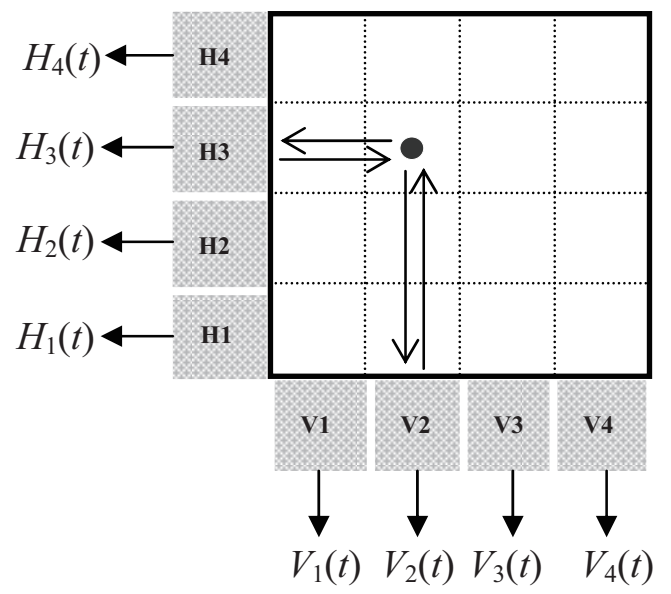

Fig. 1. Acquisition system.

maintain the temporal information. In addition, the wavelet packets permit a more flexible spectral division, allowing calculation of only the bands of interest and with the same spectral width. The registers corresponding to the higher energy $L$ frequency bands are selected from the UWPT decomposition. This results in $2 N L$ registers whose nomenclature is derived from Eq. (1) including the number of the frequency band.

$$
X_{I}(\text { band }, t)
$$

where band is the band number $(1 \ldots L)$.

The next step involves the denoising. Once the A-scan wavelet coefficients are obtained in the $L$ frequency bands, a denoising process using thresholding will be applied to each of the $2 N L$ registers obtained in the previous step. The new denoised registers are called:

$$
X_{I D}(\text { band }, t)
$$

A fundamental and difficult aspect of the process is matching the information contained in the $2 N L$ denoised registers, $X_{I D}($ band, $t)$, corresponding to the two perpendicular transducers that illuminate the same area. The matching is one of the most sensitive steps of the process and is performed using the times of flight. In order to reduce the matching sensitivity, the envelopes of $X_{I D}\left(\right.$ band,t)eX $e X_{I D}($ band, $t)$ are previously calculated. Envelopes reduce the effects of possible inaccuracies in the matching process, generating 2D-displays less sensitive to matching errors. Figure 2 shows the digital signal processing steps involved in the preparation of the ultrasonic registers to matching. The values to match for a generic coordinate $(x, y)$ are:

$$
\left[e H_{I D 1}(\text { band, } 2 x / c), e V_{I D 2}(\text { band, } 2 y / c)\right]
$$

where $e H_{I D 1}$ and $e V_{I D 2}$ are the envelopes of the registers corresponding to the transducers that illuminate the point $(x, y)$ and $c$ denotes the ultrasonic propagation velocity in the tissue. It is assumed that the origin of the coordinates is located in the bottom left hand corner of the tissue diagnosed.

Once the information for each point and each frequency is matched, the first combination can be performed. This combination is carried out independently for each of the frequency bands; the output from this step is $L 2 \mathrm{D}$ displays, one for each frequency band used. The combination of each pair of values expressed in Eq. (4) can be performed in different ways, however, previous papers [21,22] recommend 


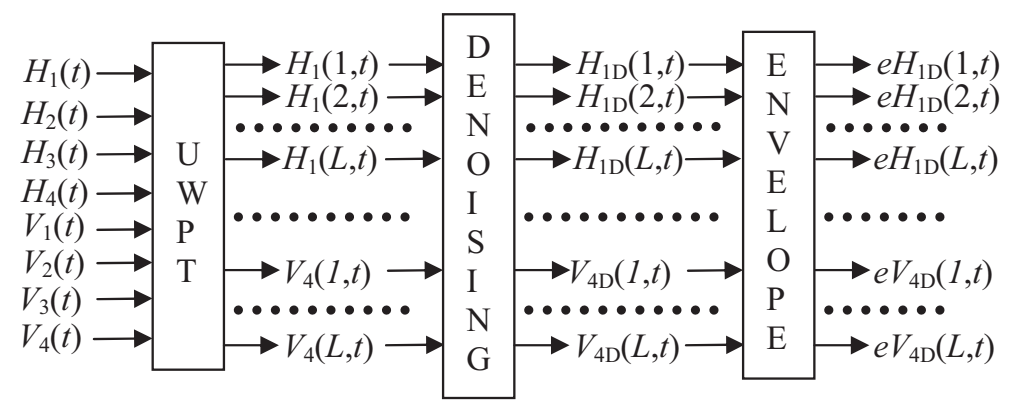

Fig. 2. Digital Signal Processing, part I: preparation of the registers to be combined.

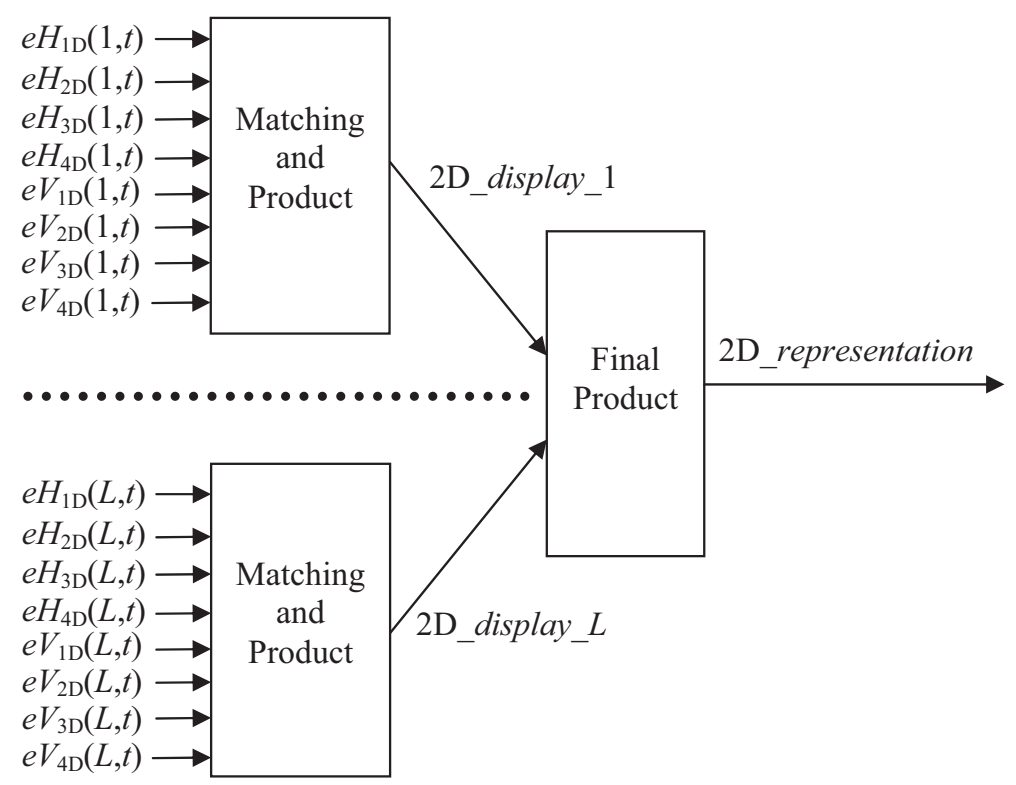

Fig. 3. Digital Signal Processing, part II: combination stages.

using a product operator to make this combination. The 2D display for each frequency band can be obtained from the following expression:

$$
2 D \_d i s p l a y \_b a n d(x, y)=e H_{I D 1}(\text { band }, 2 x / c) . e V_{I D 2}(\text { band }, 2 y / c)
$$

The $L$ 2D displays should be combined to obtain the final 2D representation. Once again, the option chosen to combine the $L$ 2D displays is by multiplying the values of the $L$ points that correspond to the same pair of coordinates $(x, y)$ in the $L 2 \mathrm{D}$ displays. Figure 3 shows a schematic representation of the digital signal processing that includes the two combination steps performed. The last combination step can be described mathematically as:

$$
2 \mathrm{D} \_ \text {representation }(x, y)=\prod_{\text {band }=1}^{L} \text { 2D_display_band }(x, y)
$$




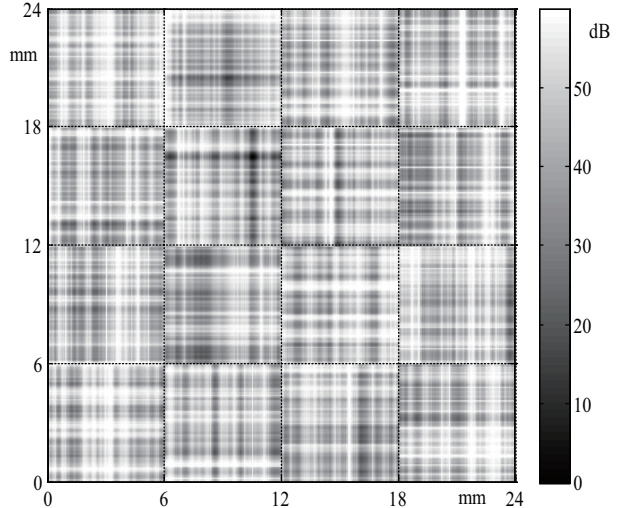

(a) A-scans $\mathrm{SNR}=4 \mathrm{~dB}$ without denoising

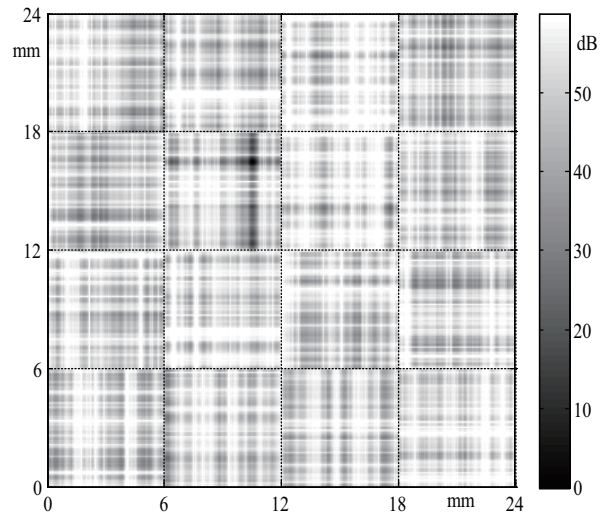

(c) A-scans $\mathrm{SNR}=6 \mathrm{~dB}$ without denoising

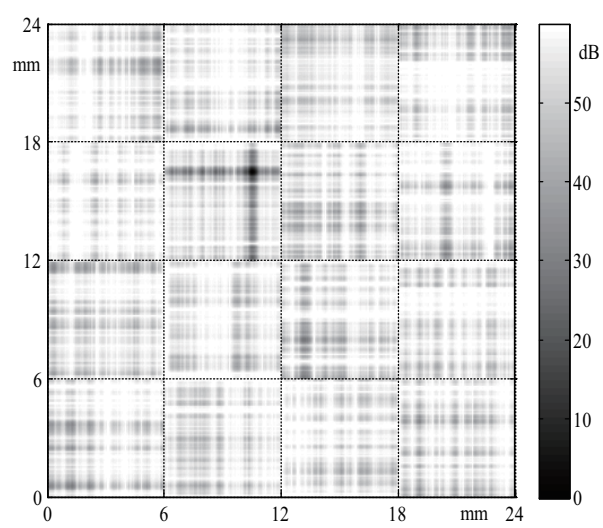

(e) A-scans SNR $=8 \mathrm{~dB}$ without denoising

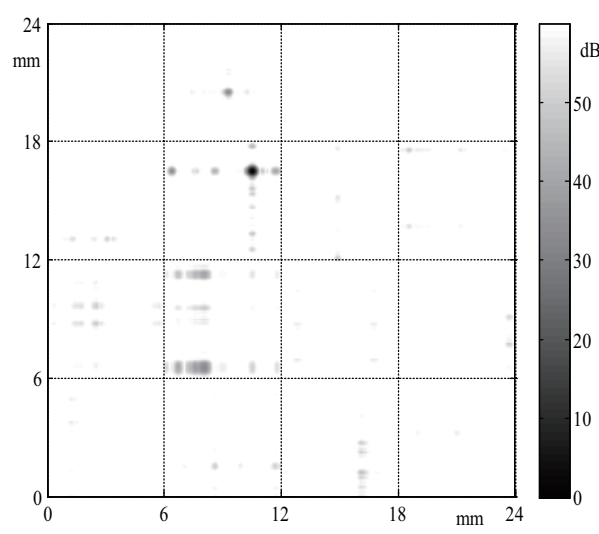

(b) A-scans $\mathrm{SNR}=4 \mathrm{~dB}$ with denoising

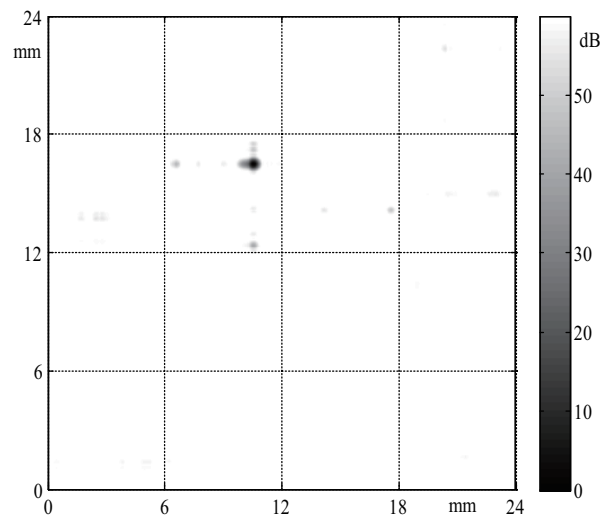

(d) A-scans SNR $=6 \mathrm{~dB}$ with denoising

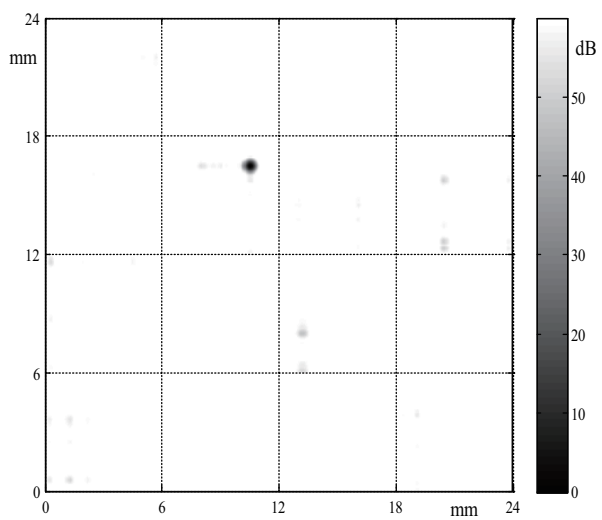

(f) A-scans $\mathrm{SNR}=8 \mathrm{~dB}$ with denoising

Fig. 4. 2D representations obtained from 8 A-scans with different SNRs. 


\section{Experimental results}

The experiments involved an ultrasonic system comprised by two perpendicular arrays of four transducers, as shown in Fig. 1. All the transducers had square surfaces with sides of $6 \mathrm{~mm}$. The total length of each array was therefore $24 \mathrm{~mm}$, so the area of tissue that could be scanned was $24 \mathrm{~mm} \times 24 \mathrm{~mm}$. An anomalous point was added in the tissue at coordinate $(11 \mathrm{~mm}, 17 \mathrm{~mm})$ in a location similar to that represented in Fig. 1.

In order to simulate the A-scans corresponding to the previous measuring scheme, two types of Ascans were generated. The first type corresponded to transducers $H_{1}, H_{2}, H_{4}, V_{1}, V_{3}$, and $V_{4}$ which only received noise reflected back from the tissue. The second type of A-scan corresponded to the transducers $H_{3}$, and $V_{4}$, which, in addition to the noise, received a clear ultrasonic echo coming from the anomalous point located at coordinate $(11 \mathrm{~mm}, 17 \mathrm{~mm})$. The ultrasonic echo used to simulate the reflection from the anomalous point was a real measured echo from a $5 \mathrm{MHz}$ Krautkramer transducer. This echo was delayed to simulate the time of flight corresponding to the distance between the anomalous point and each transducer. The speckle in the A-scans was simulated using a noise generator that included the shape of the echo produced by the Krautkramer transducer among its parameters. Therefore, the spectrums of ultrasonic echo and noise coincided. Different SNRs were taken into account in the A-scans generation, $1540 \mathrm{~m} / \mathrm{s}$ was used as ultrasonic propagation velocity and the sampling frequency was $100 \mathrm{MHz}$.

The A-scans were combined using the method based on the UWPT as described in Ref. [21]. The mother wavelet used was Daubechies 4, the maximum level of wavelet decomposition was 4 and only the two bands with the highest frequencies were selected for the combination. The denoising process was performed using soft thresholding with a SURE threshold [23].

Several sets of A-scans with different SNRs were generated. For each set of A-scans two combinations were performed: the first one without the denoising step and the second one with the denoising step. Thus, two 2D representations were obtained for each set of inputs. The 2D representations performed including the denoising process showed the best quality in all the cases. Figures $4 \mathrm{a}$ and $4 \mathrm{~b}$ show the 2D representations obtained from very noisy A-scans, with initial SNR of $4 \mathrm{~dB}$. Figures $4 \mathrm{c}$ and $4 \mathrm{~d}$ show the results after the combination of A-scans with a SNR equal to $6 \mathrm{~dB}$. Figures $4 \mathrm{e}$ and $4 \mathrm{f}$ were performed using A-scans with an initial SNR of $8 \mathrm{~dB}$. Figure 4 shows the best 2D-representations in the right-hand column in all the pairs that correspond to the column with denoising.

The 2D representations in Fig. 4 show a high resolution of $60 \mathrm{~dB}$. The anomalous point is visible at the relevant coordinate $(11 \mathrm{~mm}, 17 \mathrm{~mm})$ in all cases; the difference among the cases is in the speckle associated with each of the 2D representations. As can be seen, the speckle gradually decreases when the initial SNR of the A-scans increases. In addition, a notable improvement can be observed when the wavelet denoising process is applied during the combination, confirming the soundness of the proposed method. The results presented in Fig. 4 confirm that the denoising process produces a great improvement in the quality of the $2 \mathrm{D}$ representations.

\section{Conclusion}

Ultrasonic transducer arrays in medicine are widely used in many types of diagnosis. The majority of the arrays are linear and contain a high number of transducers in the same plane. However, this study used linear arrays that included a small number of transducers distributed in perpendicular planes. This arrangement allows diagnosis to take place using information acquired from the two different planes, 
but requires digital signal processing to allow the combination and interpretation of this complementary information.

Previous papers have studied different digital signal processing techniques for the combination of the A-scans generated by each of the transducers. The technique used in this paper is based on the use of the wavelet transform for both combination and denoising during the combination, thereby generating $2 \mathrm{D}$ representations with much higher quality.

The experiments presented in this paper show that the 2D representations obtained from noisy A-scans improve considerably with the inclusion of a denoising stage during the process. The improvement is confirmed in several cases with different levels of noise.

\section{Acknowledgements}

This work was supported by Spanish Government MEC Project TIN2013-47272-C2-1-R and by ITACA (Universitat Politècnica de València).

\section{References}

[1] R.L. Galloway, B.A. McDermott, F.L. Thurstone, A frequency diversity process for speckle reduction in real-time ultrasonic images, IEEE Transactions Ultrasonic, Ferroelectric and Frequency Control 35 (1988), 45-49.

[2] C.P. Loizou, C.S. Pattichis, et al., Comparative Evaluation of Despeckle Filtering In Ultrasound Imaging of the Carotid Artery, IEEE Transactions Ultrasonic, Ferroelectric and Frequency Control 52 (2005), 1653-1669.

[3] J. Zhanga, G. Lina, L. Wua, C. Wanga, Y. Cheng, Wavelet and fast bilateral filter based de-speckling method for medical ultrasound images, Biomedical Signal Processing and Control 18 (2015), 1-10.

[4] X. Fu, Y. Wang, L. Chen, J. Tian, An image despeckling approach using quantum-inspired statistics in dual-tree complex wavelet domain, Biomedical Signal Processing and Control 18 (2015), 30-35.

[5] S. Dahdouh, M. Dubois, et al., A 1D wavelet filtering for ultrasoundimages despeckling. Proc of SPIE 7629 (2010), 1-9, DOI: $10.1117 / 12.844388$

[6] K. Nakayama, W. Ohyama, et al., Speckle reduction of echocardiogramsvia wavelet shrinkage of ultrasonic RF signals. 13th ICBME 23 (2009), 395-398.

[7] Y. Zhang, Y.Y. Wang, et al., Doppler ultrasound signal denoisingbased on wavelet frames, IEEE Transactions Ultrasonic, Ferroelectric and Frequency Control 48 (2001), 709-716.

[8] B. Liu, Y. Wang, W. Wang, Spectrogram enhancement algorithm: A soft thresholding-based approach, Ultrasound Med Biol 25 (1999), 839-846.

[9] D.L. Donoho, I.M. Johnstone, Ideal spatial adaptation by waveletshrinkage, Biometrika 81 (1994), 425-455

[10] D.L. Donoho, I.M. Johnstone, et al., Wavelet shrinkage: Asymptotia, J R Stat Soc B 57 (1995), 301-369.

[11] D.L. Donoho, I.M. Johnstone, Adapting to unknown smoothnessvia wavelet shrinkage, J Am Stat Assoc 90 (1995), 1200-1224.

[12] F. Wang, Z. Ji, Application of the Dual-tree ComplexWaveletTransform in Biomedical Signal Denoising, Bio-Medical Materials and Engineering 24 (2014), 109-115; DOI: 10.3233/BME-130790.

[13] J.L. San Emeterio, M.A. Rodriguez-Hernandez, Wavelet denoising of ultrasonic A-scans for detection of weak signals, 19th International Conference on Systems, Signals and Image Processing (IWSSIP), Vienna, Austria (2012), 54-57.

[14] R.R. Coifman, D.L. Donoho, Translation-invariant de-noising, Lect Notes Stat 103 (1995), 125-150.

[15] E. Pardo, J.L. San Emeterio, et al., Shift invariant wavelet denoisingof ultrasonic traces, ActaAcust United Ac 94 (2008), 685-693.

[16] J.L. San Emeterio, M.A. Rodriguez-Hernandez, Wavelet Cycle Spinning Denoising of NDE Ultrasonic Signals Using a Random Selection of Shifts, Journal Nondestructive Evalaluation 34 (2015), 270, DOI: 10.1007/s10921-014-0270-8

[17] M.A. Rodriguez-Hernandez, J.L. San Emeterio, Noise Reduction Using Wavelet Cycle Spinning. Analysis of Useful 189 Periodicities in the z-Transform Domain, Signal Image Video Processing, 10 (2016), 519-526; DOI: 10.1007/s11760015-0762-8.

[18] A.W. Meyer, J.V. Candy, Iterative processing of ultrasonic measurements to characterize flaws in critical optical components IEEE Trans. Ultrason. Ferroelect. Freq. Control 8 (2002), 1124-1138. 
[19] D. Reguieg, F. Padilla, et al., Ultrasonic transmission device based on crossed beam forming, Proc. IEEE Ultrasonics Symp. (2006), 2108-2111.

[20] H.J. Zhu, P. Yang, T.B. Yao, 3D reconstruction of ultrasound scanned data for tissue mimicking material sample, BioMedical Materials and Engineering 24 (2014), 2771-2781, DOI: 10.3233/BME-141095.

[21] M.A. Rodriguez-Hernandez, A. Ramos, J.L. San Emeterio, Wavelet-based 2D fusing of ultrasonic pulse-echo traces measured from two arrays radiating orthogonal beams, Measurement Science and Technology 22 (2011), 1-14, DOI: 10.1088/0957-0233/22/10/105701

[22] M.A. Rodriguez-Hernandez, A. Ramos, J.L. San Emeterio, Localization of Isolate Flaws by Combination of Noised Signals Detected from Perpendicular Transducers, NDT and E International 37 (2004), 345-352.

[23] D.L. Donoho, De-noising by soft thresholding, IEEE Transactions on Information Theory 41 (1995), 613-627. 